defined by a 50 point reduction in IBS-SSS. Data, expressed as mean \pm standard error, were compared statistically before and after treatment using paired t-tests.

Results Young patients fulfilling Rome III diagnostic criteria for IBS $(n=26$, median age 16 (range -8$)$ years, $n=17(65 \%)$ female, mean duration of IBS $5.3 \pm 0.9$ years, $n=11$ IBS-D, $n=6$ IBS-C and $n=9$ IBS-mixed) completed the hypnotherapy programme. Mean baseline IBS-SSS was $321.5 \pm 16.0$. After hypnotherapy, $n=23 / 26 \quad(88 \%)$ responded, with an overall mean reduction in IBS-SSS of $-160.9 \pm 15.4(P<0.0001)$, and $n=19 / 26(73 \%)$ achieved the FDA recommended outcome of $\geq 30 \%$ reduction in abdominal pain scores. Hypnotherapy also improved; mean non-colonic symptom score by $102.1 \pm 15.0$ $(P<0.0001)$, mean HADS-anxiety by $-3.0 \pm 0.8 \quad(P=0.0007)$, mean HADS-depression by $-2.1 \pm 0.6 \quad(P=0.002)$, and improved mean QoL score by $+89.7 \pm 13.1(P<0.0001)$.

Conclusion These data, which form one of the largest reported series of gut-focussed hypnotherapy in children and adolescents with severe IBS, suggest that this treatment is even more effective in this group of patients than in adults. Hypnotherapy in severe childhood IBS patients may therefore have a role in preventing further suffering in adult life, reducing healthcare utilisation and related costs with wider socioeconomic benefits. Furthermore, it allows many of them to return to full time education.

\section{OWE-10 COGNITIVE BEHAVIOURAL THERAPY FOR IRRITABLE BOWEL SYNDROME: 24 MONTH FOLLOW-UP OF ACTIB TRIAL PARTICIPANTS}

${ }^{1}$ Assoc Hazel Everitt* ${ }^{2}$ Sabine Landau, ${ }^{2}$ Alice Sibelli, ${ }^{1}$ Stephanie Hughes, ${ }^{1}$ Gillian O'Reily, ${ }^{2}$ Sula Windgassen, ${ }^{2}$ Rachel Holland, ${ }^{1}$ Paul Little, ${ }^{2}$ Paul McCrone, ${ }^{1}$ Felicity Bishop, ${ }^{2}$ Kim Goldsmith, ${ }^{3}$ Nicholas Coleman, ${ }^{4}$ Robert Logan, ${ }^{2}$ Trudie Chlader, ${ }^{2}$ Rona Moss-Morris. ${ }^{1}$ University Of Southampton, Southampton, UK; ${ }^{2}$ Kings College , London, UK; ${ }^{3}$ Southampton University Hospital, Southampton, UK; ${ }^{4}$ Kings College Hospital, London, UK

10.1136/gutjnl-2019-BSGAbstracts.389

Introduction The ACTIB (Assessing Cognitive behavioural Therapy (CBT) for IBS) randomised controlled trial $(n=558)$ was a 3 arm multicentre trial which showed that telephone therapist-delivered CBT (TCBT) and web-based CBT (WCBT) with minimal therapist support were significantly more effective than treatment as usual (TAU) at reducing IBS symptom severity and impact at 12 months in adults with refractory IBS.

Methods A 24 month naturalistic follow-up of ACTIB participants. Participants were recruited from 74 primary care general practice (GP) surgeries and 3 secondary care gastroenterology outpatient clinics in the South of England and London, May 2014 to March 2016. 24 month data collection completed May 2018. TAU participants were given access to the WCBT website from 12 months. Co-primary outcome measures (IBS Symptom Severity Score (IBS SSS) and Work and Social Adjustment Scale (WSAS). Formal trial arm comparisons were Intention-to-treat analyses by multiple imputation to account for missing data.

Results $57.9 \%$ (323/558) of participants randomised were followed up to 24 months. Only 10 TAU participants chose to access WCBT.

Preliminary results Compared to TAU (IBS SSS score 198 at 24 months), IBS SSS scores were 40.5 (95\% CI $(15.0$ to 66.0)) points lower $(\mathrm{p}<0.002)$ in TCBT and 12.9 (95\% CI 12.9 to 38.8$)$ points lower $(\mathrm{p}=0 \cdot 3)$ in WCBT at 24 months.
Assessing IBS-SSS responders (participants with a clinically significant IBS SSS change ( $\geqq 50$ point) from baseline to 24 months: $84 / 119$ (70.6\%) were responders in TCBT, 62/99 $(62.6 \%)$ in WCBT and 48/105 (45.7\%) in TAU.Compared to TAU (WSAS score 7.6 at 24 months) WSAS was 3.1 (95\% CI 1.3 to 4.9$)$ points lower $(\mathrm{p}<0 \cdot 001)$ in TCBT and $1.9(95 \%$ CI 0.1 to 3.7$)$ points lower $(\mathrm{p}<0 \cdot 04)$ in WCBT. Patient enablement (responders): TCBT compared to TAU OR 8.3 (95\% CI 4.2 to 16.4$) \mathrm{p}<0 \cdot 001)$, WCBT to TAU OR $3.3(95 \% \mathrm{CI}$ 1.8 to 6.0$) \mathrm{p}=0.001$; Hospital anxiety and depression scale (HADS) TCBT to TAU 3.1 (95\% CI 1.6 to 4.7$) \mathrm{p}<0.001$ and WCBT to TAU (95\% CI 2.7 (1.0 to 4.4$) \mathrm{p}=0.002$.

Conclusions At 24 months sustained benefits were seen in both CBT groups compared to TAU, particularly on impact of IBS symptoms. Some previous gains were reduced compared to 12 month follow-up in the intention-to-treat analysis. Complete case analysis indicated those who had adhered to CBT treatments maintained large clinically significant gains in both symptoms and impact at 24 months. Increasing access to CBT for IBS could achieve long term-benefits for patients

\section{OWE-11 THE PREVALENCE AND BURDEN OF ROME IV FUNCTIONAL COLORECTAL DISORDERS IN ULCERATIVE COLITIS}

${ }^{1}$ Gaurav B Nigam*, 'Jimmy K Limdi, ${ }^{2}$ Shaheen Hamdy, ${ }^{3}$ Dipesh H Vasant. ${ }^{1}$ Pennine Acute Hospitals; ${ }^{2}$ Salford Royal Foundation Trust; ${ }^{3}$ Manchester University Foundation Trust

\subsection{6/gutjnl-2019-BSGAbstracts.390}

Introduction Despite advances in Ulcerative Colitis (UC) therapies, many patients suffer refractory symptoms in the absence of active inflammation. For this group, treatment remains challenging, with a paucity of therapeutic options. In this prospective, ongoing study, we aim to determine the prevalence and burden of functional colorectal disorders in patients with quiescent UC using validated questionnaires.

Methods In a cross-sectional study, consecutive patients with UC attending Inflammatory Bowel Disease (IBD) clinics were invited to participate. Patients completed a series of validated questionnaires; including Hospital Anxiety and Depression Scale (HADS), the Rome IV diagnostic questionnaire for functional gastrointestinal disorders (FGIDs), an IBD-QoL score and the IBD-control questionnaire. Participants were requested to return a Faecal Calprotectin (FCP) within 2 weeks of completing questionnaires. Quiescent UC was defined as IBD-control 8 score $\geq 13$ and IBD-control-VAS $\geq 85$, and/or FCP levels $\leq 250$ (where available, FCP data were used in preference to IBD-control to classify UC activity). Based on Rome IV diagnosis and UC disease activity (active or quiescent), patients were divided into groups and data compared using non-parametric tests.

Results Overall, $\mathrm{n}=97$ UC patients $(\mathrm{n}=50$ males, mean age 48 (range 1-2)) participated. 41/97, (42\%) UC patients met the Rome IV diagnostic criteria for $\geq 1$ FGIDs (irritable bowel syndrome $n=26$, functional constipation $n=6$ and faecal incontinence $(\mathrm{FI}) \mathrm{n}=22$ ). Disease activity data (IBD-control and/or FCP) were available for all patients, and based on these $61 / 97,63 \%$ had quiescent UC. Within the quiescent UC group, 25/61 (41\%) met the Rome IV diagnostic criteria for $\geq 1$ FGIDs (irritable bowel syndrome $n=14$, functional constipation $n=3$ and FI $n=13$ ). Within the active UC group, those with co-existing FGIDs, compared to those without FGIDs, 